\title{
The PANDORA project: an experimental setup for measuring in-plasma $\beta$-decays of astrophysical interest
}

\author{
David Mascali ${ }^{1}$, Maurizio Busso ${ }^{2}$, Alberto Mengoni ${ }^{3,4}$, Simone Amaducci ${ }^{1,5}$, Giuseppe \\ Castro $^{1}$, Luigi Celona ${ }^{1}$, Gianluigi Cosentino ${ }^{1}$, Sergio Cristallo ${ }^{2}$, Paolo Finocchiaro ${ }^{1}$, Alessio \\ Galatà ${ }^{6}$, Santo Gammino ${ }^{1}$, Cristian Massimi ${ }^{4}$, Mario Maggiore ${ }^{6}$, Giorgio Mauro ${ }^{1}$, Maria \\ Mazzaglia ${ }^{1}$, Eugenia Naselli1 ${ }^{1,5}$, Fabrizio Odorici $^{4}$, Sara Palmerini $^{2}$, Domenico \\ Santonocito ${ }^{1}$, Giuseppe Torrisi ${ }^{1}$
}

${ }^{1}$ INFN-Laboratori Nazionali del Sud, Catania, Italy

${ }^{2}$ INFN, Sezione di Perugia, Perugia, Italy

${ }^{3}$ ENEA, Bologna, Italy

${ }^{4}$ INFN, Sezione di Bologna, Italy

${ }^{5}$ Università degli Studi di Catania, Catania, Italy

${ }^{6}$ INFN-Laboratori Nazionali di Legnaro, Legnaro (PD), Italy

\begin{abstract}
Experiments performed on Storage Rings have shown that lifetimes of beta-radionuclides can change dramatically as a function of the ionization state. PANDORA (Plasmas for Astrophysics, Nuclear Decay Observation and Radiation for Archaeometry) aims at measuring, for the first time, nuclear $\beta$-decay rates in stellar-like conditions, especially for radionuclides involved in nuclear-astrophysics processes (BBN, sprocessing, CosmoChronometers, Early Solar System formation). Compact magnetic plasma traps, where plasmas reach density $\mathrm{n}_{\mathrm{e}} \sim 10^{11}-10^{14} \mathrm{~cm}^{-3}$, and temperature $T_{\mathrm{e}} \sim 0.1-30 \mathrm{keV}$, are suitable for such studies. The decay rates can be measured as a function of the charge state distribution of the inplasma ions. The collaboration is now designing the plasma trap able to reach the needed plasma densities, temperatures and charge states distributions. A first list of radioisotopes, including tens of physics cases of potential interest is now available. Possible physics cases include, among the others, ${ }^{204} \mathrm{Tl},{ }^{63} \mathrm{Ni},{ }^{60} \mathrm{Co},{ }^{171} \mathrm{Tm},{ }^{147} \mathrm{Pm},{ }^{85} \mathrm{Kr},{ }^{176} \mathrm{Lu}$ and the pairs ${ }^{187} \mathrm{Re}-$ ${ }^{187} \mathrm{Os}$ and ${ }^{87} \mathrm{Sr}-{ }^{87} \mathrm{Rb}$, which play a crucial role as cosmo-clock. Physics cases are now under evaluation in terms of lifetime measurements feasibility in a plasma trap.
\end{abstract}




\section{Introduction}

In the last decades, there was a growing interest in Nuclear Astrophysics involving lowenergy heavy-ion reactions and radioactive-decays in astrophysical environment.

Terrestrial measurements involve, up to now, neutral species, while both nuclear reactions and decays involved in nuclear astrophysics should be investigated in peculiar plasma environments. Indeed, plasma is a quite common state of matter in our universe, having properties unlike those of the other states.

There is great interest in Nuclear Astrophysics for any attempt at providing improved knowledge of weak interactions in physical conditions similar to those of nucleosynthesis environments (stars, the Big Bang and cosmic rays). In particular, large modifications of nuclear half-lives with respect to neutral atoms have been predicted and observed in beta decays from highly charged ions $[1,2]$, a situation that up to now has been experimentally studied solely at ion storage rings. Here we propose a new experimental scenario, i.e. the decay study in a magnetically confined plasma: we will outline briefly the most relevant scientific cases, then emphasizing a few of them for which the PANDORA experiment will provide significant new insight.

\subsection{Plasma properties relevant for astrophysics}

In general, in order to estimate the detailed nucleosynthetic consequences of "slow" neutron fluxes occurring in various phases of stellar evolution, one would want to know the halflives of all the radioactive nuclei sited at possible branching points along the $n$-capture path, in thermal conditions spanning the range from $8 \mathrm{keV}$ (the energy at which neutrons are released by the ${ }^{13} \mathrm{C}(\alpha, n){ }^{16} \mathrm{O}$ reaction in low mass AGB stars [3, 4]) to above $90 \mathrm{keV}$ (the typical thermal energy of C-burning shell phases in massive-star evolution [5]; in this last environment, the isotope ${ }^{22} \mathrm{Ne}$, remaining after core He burning, can generate bursts of neutrons through the ${ }^{22} \mathrm{Ne}(\alpha, n)^{25} \mathrm{Mg}$ reaction [6, 7]). In between these extreme cases, neutron fluxes from the ${ }^{22} \mathrm{Ne}$ source can occur around $18-21 \mathrm{keV}[6,7]$ (core He-burning stages in massive stars) and at $23-30 \mathrm{keV}[4,8]$ (thermal instabilities of the He-burning shell in low and intermediate mass stars). Attention must today be paid also to the complementary neutron source ${ }^{12} \mathrm{C}\left({ }^{12} \mathrm{C}, n\right){ }^{23} \mathrm{Mg}$ at around $100 \mathrm{keV}$ [9], whose effectiveness in advanced stages of massive star nucleosynthesis was recently re-emphasized by new measurements $[9,10]$.

\subsubsection{The use of laboratory plasmas for $\beta$-decays measurements}

Compact magnetic traps have been mostly used as ion sources since '70: such traps (called "B-minimum" machines, from the peculiar shape of the magnetic field) have supported the growing request of intense beams of multicharged ions coming from both fundamental science (nuclear and particle Physics especially) and applied research (neutrons spallation sources, subcritical nuclear reactors, hadrotherapy facilities, material treatments, ion implantation).

Inside these machines a dense and hot plasma - made of multicharged ions immersed in a dense cloud of energetic electrons - is confined by multi-Tesla magnetic fields and resonantly heated by some $\mathrm{kWs}$ of microwave power in the $2.45-28 \mathrm{GHz}$ frequency range. The plasmas reach $\mathrm{n}_{\mathrm{e}} \sim 10^{11}-10^{13} \mathrm{~cm}^{-3}, \mathrm{~T}_{\mathrm{e}} \sim 0.1-100 \mathrm{keV}$ of electron density and temperature, respectively.

These plasmas are MHD stable, magnetized, living several hours or days with on average constant local density and temperature, so they represent an attractive environment for the studies envisaged in the framework of PANDORA. 
Table 1. Preliminary list of potential physics cases of astrophysical interest.

\begin{tabular}{|c|c|c|c|c|c|}
\hline Isotope & Lab $\tau_{1 / 2}(\mathrm{yr})$ & $\mathbf{J} \pi$ & $\mathbf{Z}$ & $\mathbf{E}_{\gamma}(\mathrm{keV})$ & decay \\
\hline${ }^{63} \mathrm{Ni}$ & 100.1 & $1 / 2^{-}$ & 28 & No & $\beta^{-}$ \\
\hline${ }^{79} \mathrm{Se}$ & $1.13 \times 10^{6}$ & $7 / 2^{+}$ & 34 & No & $\beta^{-}$ \\
\hline$\left({ }^{79 m} \mathrm{Se}\right.$ & $3.9 \mathrm{~m}$ & $1 / 2^{-}$ & 34 & 95.7 & IT, $\left.\beta^{-}\right)$ \\
\hline${ }^{81} \mathrm{Kr}$ & $2.29 \times 10^{5}$ & $7 / 2^{+}$ & 36 & 276 & $\varepsilon$ \\
\hline$\left({ }^{81 \mathrm{~m}} \mathrm{Kr}\right.$ & $13.1 \mathrm{~s}$ & $1 / 2^{-}$ & 36 & 190.62 & $I T, \varepsilon)$ \\
\hline${ }^{85} \mathrm{Kr}$ & 10.756 & $9 / 2+$ & 36 & $130-514$ & $\beta^{-}$ \\
\hline$\left({ }^{85 \mathrm{~m}} \mathrm{Kr}\right.$ & $4.48 \mathrm{~h}$ & $1 / 2^{-}$ & 36 & $129-731$ & $\left.I T, \beta^{-}\right)$ \\
\hline${ }^{93} \mathrm{Zr}$ & $1.5 \times 10^{6}$ & $5 / 2^{+}$ & 40 & 30.7 & $\beta^{-}$ \\
\hline${ }^{95} \mathrm{Zr}$ & $64 d$ & $5 / 2^{+}$ & 40 & $235-756$ & $\beta^{-}$ \\
\hline${ }^{99} \mathrm{Tc}$ & $2.1 \times 10^{5}$ & $9 / 2+$ & 43 & 89.6 & $\beta^{-}$ \\
\hline${ }^{106} \mathrm{Ru}(?)$ & $373.5 \mathrm{~d}$ & $0^{+}$ & 44 & $\begin{array}{l}\text { Many } \\
\left({ }^{106} \mathrm{Rh}\right)\end{array}$ & $\beta^{-}$ \\
\hline${ }^{134} \mathrm{Cs}$ & 2.065 & $4^{+}$ & 55 & $>600$ & $\beta^{-}, \varepsilon$ \\
\hline$\left({ }^{134 \mathrm{~m}} \mathrm{Cs}\right.$ & $2.903 \mathrm{~h}$ & $8^{-}$ & 55 & 138.744 & $I T$ \\
\hline${ }^{135} \mathrm{Cs}$ & $2.3 \times 10^{6}$ & $7 / 2^{+}$ & 55 & 786,846 & $\beta^{-}$ \\
\hline${ }^{137} \mathrm{Cs}$ & 30.07 & $7 / 2^{+}$ & 55 & $238-661$ & $\beta^{-}$ \\
\hline${ }^{147} \mathrm{Pm}$ & 2.6 & $7 / 2^{+}$ & 61 & 76-197 & $\beta^{-}$ \\
\hline${ }^{151} \mathrm{Sm}$ & 90 & $5 / 2^{-}$ & 62 & No & $\beta^{-}$ \\
\hline${ }^{155} \mathrm{Eu}$ & 4.7 & $5 / 2^{+}$ & 63 & 10-146 & $\beta^{-}$ \\
\hline${ }^{171} \mathrm{Tm}$ & $1.92 \mathrm{~d}$ & $1 / 2^{+}$ & 69 & 66.7 & $\beta^{-}$ \\
\hline${ }^{176} \mathrm{Lu}$ & $3.78 \times 10^{10}$ & $7^{-}$ & 71 & $88-400$ & $\beta^{-}$ \\
\hline$\left({ }^{176 m} \mathrm{Lu}\right.$ & $3.635 \mathrm{~h}$ & $1^{-}$ & 71 & $82-1237$ & $\left.\beta^{-}, \varepsilon\right)$ \\
\hline${ }^{204} \mathrm{Tl}$ & 5.45 & $2^{-}$ & 81 & $511 \varepsilon^{+} \mathrm{e}^{-}$ & $\beta^{-} \varepsilon+\beta^{+}$ \\
\hline${ }^{94} \mathrm{Nb}$ & $2.03 \times 10^{4}$ & $6^{+}$ & 41 & $702-871$ & $\beta^{-}$ \\
\hline${ }^{93} \mathrm{Mo}$ & $4.0 \times 10^{3}$ & $5 / 2^{+}$ & 42 & 30.7 & $\varepsilon$ \\
\hline${ }^{129} \mathrm{I}$ & $1.57 \times 10^{7}$ & $7 / 2^{+}$ & 53 & 40 & $\beta^{-}$ \\
\hline${ }^{205} \mathrm{Tl}$ & stable & $1 / 2^{+}$ & 81 & No & if ion $\beta^{-}$ \\
\hline${ }^{163} \mathrm{Dy}$ & stable & $5 / 2^{-}$ & 66 & $77.5(\mathrm{Ho})$ & if $10 v . \beta$ \\
\hline
\end{tabular}

\section{Relevant Physics Cases}

In table 1, a list of isotopes that play an important role in nucleosynthesis networks and are potentially suitable to be studied in laboratory is given. This relatively short list is taken from a longer one, that in principle contains more than 120 isotopes.

In some cases (e.g. ${ }^{163} \mathrm{Dy}$, or $\left.{ }^{205} \mathrm{Tl}\right)$ the nuclides are stable in terrestrial laboratories, but become unstable when they are ionized in a hot stellar plasma. Due to the large number of different situations and nuclear paths near the valley of $\beta$-stability, only a limited subset of cases will be considered here.

The choice is to start from isotopes for which: i) samples can be found rather easily; ii) $\gamma$ rays in a suitable energy interval for the ion trap PANDORA are emitted by the daughter nuclei, or alternative measuring techniques can be safely envisaged; iii) lifetimes are in a range such that in a reference scheme like that of Figure 1, the experimental conditions for detection in the instrument are guaranteed. 


\subsection{Selection of the first three cases for PANDORA - phase 1}

We adopted a selection procedure for sorting out - from the list of tens of radioisotopes reported in table 1 - three cases for the first run of measurements. The selection procedure took into account a combination of four "ratings", concerning:

(i) Scientific relevance (importance for astrophysics)

(ii) Expected variation of the lifetime (with the criterion: the higher-the better, in order to have the clearest experimental evidence)

(iii) Impact of the isotope characteristics on the trap design (needed ionisation state, etc.)

(iv) Type of element (gas? Metal? Rare isotope?)

The selection procedure has finally given as output three isotopes: ${ }^{176} \mathrm{Lu}$ (is it a cosmothermometer or a cosmo-chronometer?), ${ }^{134} \mathrm{Cs}$ (relevant for the production of the s-only isotopes ${ }^{134} \mathrm{Ba}$ and ${ }^{136} \mathrm{Ba}$ ), and ${ }^{94} \mathrm{Nb}$ (relevant for the abundance of ${ }^{94} \mathrm{Mo}$ in single or binary systems of stars).

Table 2. First isotopes selected for in-plasma decay time measurements

$\begin{array}{ccl}\text { Isotope } & \mathrm{T}_{1 / 2}(\mathrm{yr}) & \text { Ey }(\mathrm{keV}) \\ { }^{176} \mathrm{Lu} & 3.78 \times 10^{10} & 88-400 \\ { }^{134} \mathrm{Cs} & 2.06 & >600 \\ { }^{94} \mathrm{Nb} & 2.03 \times 10^{4} & >700\end{array}$

\subsubsection{The cases of ${ }^{176} \mathrm{Lu},{ }^{134} \mathrm{Cs},{ }^{94} \mathrm{Nb}$}

${ }^{176} \mathrm{Lu}$ is a very long-lived nucleus in laboratory conditions and in principle might act as a cosmo-chronometer, if not for its complex behaviour in hot stellar plasmas. Here, the sprocess branching point at ${ }^{176} \mathrm{Lu}$ is among the most important ones for the precise understanding of slow neutron captures in the AGB phases of low and intermediate mass stars, as it determines the abundance of ${ }^{176} \mathrm{Hf}$, an "s-only" nucleus, shielded against fast decays from the r-process by ${ }^{176} \mathrm{Lu}$ itself (if it remains long-lived) and/or by ${ }^{176} \mathrm{Yb}$ (see Figure 2). A discussion of the temperature and density dependence of ${ }^{176} \mathrm{Lu}$ decay was presented theoretically in [11,12], but assuming Local Thermodynamic Equilibrium (LTE) and with a somewhat simplified approach. A direct measurement would be therefore extremely interesting for stellar nucleosynthesis and appears to be feasible by Pandora. It would also clarify whether ${ }^{176} \mathrm{Lu}$ can be still considered as a chronometer, or has instead to be regarded as a thermometer of the s-processing site (this last being the current interpretation prevailing today).

Figure 1 reports about expectations of ${ }^{17} \mathrm{Lu}$ lifetime variation as a function of the plasma temperature (namely, of its charge state distribution). Under terrestrial conditions, the lifetime was measured as order of magnitude of $10^{10}$ years. In [2] authors determined a collapse of several order of magnitude from theoretical calculations, at temperatures above 2-3 keVs. Calculations have been now repeated by applying the FLYCHK code considering LTE (local thermal equilibrium) approximation for the plasma electrons, being in reasonable agreement with the previous ones. Despite this assumption is questionable, in low density plasmas, calculations performed by the FLYCHK code say that for most of the selected cases the LTE and non-LTE scenarios do not differ hugely each other. 


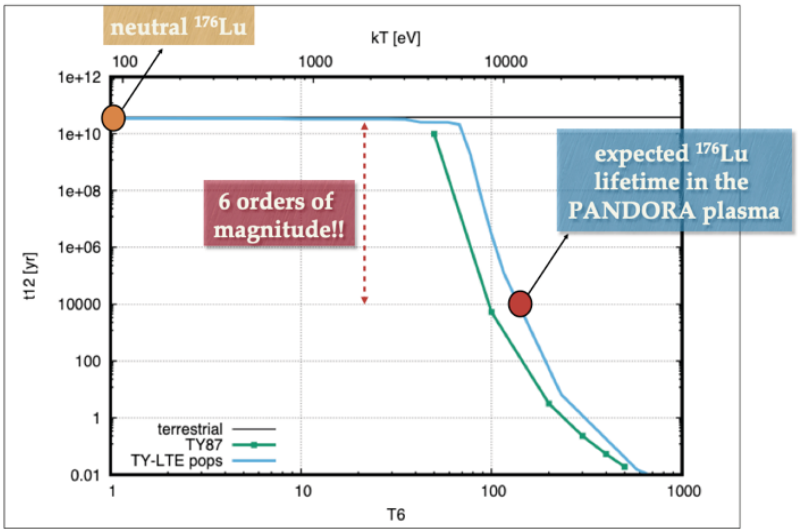

Fig. 1. Expected ${ }^{176} \mathrm{Lu}$ Lifetime vs. plasma temperature trend at densities of PANDORA's plasmas

Work is in progress and further, more detailed calculations will be needed and performed in the near future.

A scheme of the reaction branching occurring at ${ }^{134} \mathrm{Cs}$ in s-process situations is shown in Figure 2, taken from reference [3].

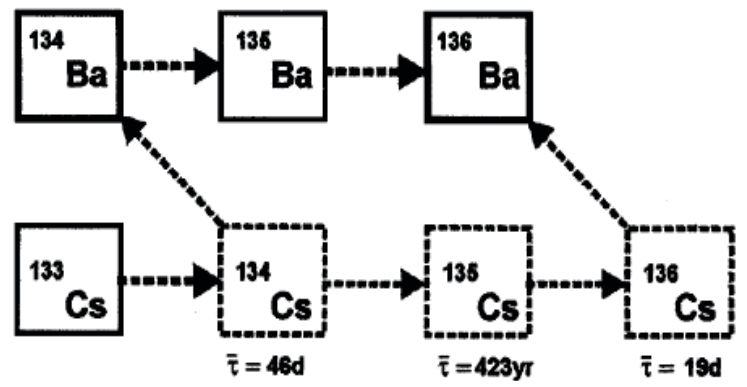

Fig. 2. Scheme of s-process branching occurring for ${ }^{134} \mathrm{Cs}$.

According to theoretical predictions [2] ${ }^{134} \mathrm{Cs}$ lifetime is reduced to 48 days at $\mathrm{T}=3 \times 10^{8} \mathrm{~K}$ (representing typical conditions in thermal pulses of TP-AGB stars of low mass). This datum forbids the proper reproduction of the two s-only isotopes ${ }^{134} \mathrm{Ba},{ }^{136} \mathrm{Ba}$ in suitable proportions. This impossibility is known to have a nuclear, not a stellar origin. A longer half-life of ${ }^{134} \mathrm{Cs}$ in stellar conditions, or a more efficient neutron-capture process (larger cross section) of ${ }^{134} \mathrm{Cs}$ itself could solve the puzzle.

${ }^{94} \mathrm{Nb}$ is the main production channel for ${ }^{94} \mathrm{Mo}$, shielded from the fast decays of the rprocess. ${ }^{94} \mathrm{Nb}$ comes indirectly from ${ }^{93} \mathrm{Zr}$, which actually behave as stable in timescale of TP-AGB stars evolution: its presence, and thus the presence of ${ }^{94} \mathrm{Mo}$, should be strongly underabundant. However, more than $50 \%$ of AGB stars are in binary or multiple systems: mass transfer to occurs to a secondary component from a TP-AGB to a companion in RGB stage. In this complex situation, the exact contribution of s-processing to ${ }^{94} \mathrm{Mo}$ is badly determined. In presolar $\mathrm{SiC}$ grains this nucleus is underabundant and we aim at disentangling this complex scheme of processes, probably mainly in the lifetime of ${ }^{94} \mathrm{Nb}$ itself.

Both for ${ }^{94} \mathrm{Nb}$ and ${ }^{134} \mathrm{Cs}$ calculations like the ones of figure 1 can be done, showing similar lifetime collapses as the plasma temperature increases. 


\section{The design of the experimental setup}

The basic idea of PANDORA (Plasmas for Astrophysics Nuclear Decays Observation and Radiation for Archaeometry) is that compact and flexible magnetic plasma traps (where plasmas reach density $\mathrm{n}_{\mathrm{e}} \sim 10^{11}-10^{13} \mathrm{~cm}^{-3}$, and temperature $\mathrm{T}_{\mathrm{e}} \sim 0.1-100 \mathrm{keV}$ ) become the environment for measuring, for the first time, the lifetime of the above mentioned $\beta$-decay isotopes in stellar-like conditions.

The overall setup scheme is illustrated in figure 3 . It consists of 3 superconducting coils and a copper-made "monocoil" hexapole able to produce a multi-mirror confining B-field. 14 HpGe detectors should be used for tagging $\beta$-decays by the $\gamma$-rays emitted from the decayproducts in their excited states.

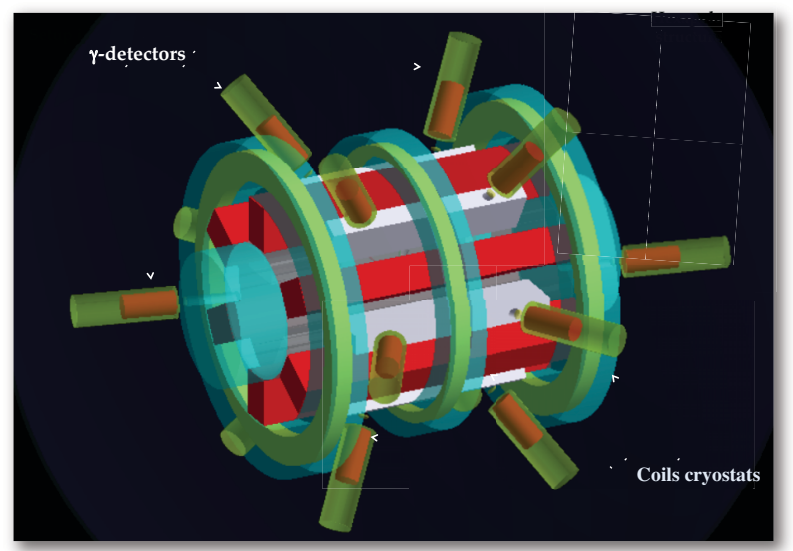

Fig. 3. Scheme of PANDORA overall setup as modelled and simulated by GEANT4 code: it consists of a multi-mirror magnetic trap surrounded by a detectors array.

Inside the magnetic trap, $\alpha$ "buffer plasma" is created by $\mathrm{He}, \mathrm{O}$ or Ar up to densities of $10^{13}$ $\mathrm{cm}^{-3}$. The isotope is then directly fluxed (if gaseous) or vaporized by appropriate ovens and then fluxed inside the chamber to be turned into plasma-state. Relative abundances of buffer vs. isotope densities range from 100:1 (if the isotope is in metal state) to 3:1 (in case of gaseous elements). The plasma is maintained in dynamical equilibrium by equalizing input fluxes of particles to losses from the magnetic confinement. In particular, the number of decays per unit of times can be written as:

$$
\frac{d N}{d t}=\lambda n_{i} V
$$

Where $\mathrm{dN} / \mathrm{dt}$ is the number of decays per unit of time, $\lambda$ is the isotope's activity, $\mathrm{n}_{\mathrm{i}}$ is the plasma ions density and $\mathrm{V}$ is the plasma volume. If the plasma is in dynamical equilibrium (i.e. fluxes of ionized particles is equal to the ones of ion losses from the trap), then the term, $\lambda \mathrm{n}_{\mathrm{i}} \mathrm{V}$ is constant in time, and the equation 1 can be easily integrated:

$$
\int_{0}^{t_{\text {meas. }}} d N=\int_{0}^{t_{\text {meas. }}} \lambda n_{i} V d t
$$




$$
N\left(T_{\text {meas }}\right)=\lambda n_{i} V_{\text {plasma }} T_{\text {meas }}
$$

Where $t_{\text {meas }}$ is the overall measurement time over which the $\gamma$-rays produced by the excited states of the decays products are counted by the detector array.

Hence, the number of measured decays scales linearly with the measurement time, provided that the dynamical equilibrium has been established. Under this assumption, the decay constant $\lambda$ is simply the proportionality constant of eq. 3 .

Equation 3 implies also that the plasma density must be known and on-line monitored all along the measurement in order to deconvolve $\lambda$.

\subsection{Design of the detectors array}

The design of the detection system has been based on GEANT4 simulations. As input for the simulations, we used the geometrical design shown in fig.3: more in details, it consists of a stainless steel chamber (radius $175 \mathrm{~mm}$, length $800 \mathrm{~mm}$ and thickness $10 \mathrm{~mm}$ ) drilled with 12 holes having a diameter of $25 \mathrm{~mm}$, that can host long collimators. The overall magnetic system consists of three NbTi superconducting coils (in green) surrounded by a cryostat each one (in light, transparent cyan), and an hexapole, in figure 3 modelled as 6 bars (in red) with iron inserts (in grey) in between.

These iron bars put in the hexapole interspaces are drilled, in order to use them as collimators and thus suppress as much as possible photons fluxes coming from the walls and not directly from the plasma core. The figure also shows an array of $14 \gamma$-detectors (length: $8 \mathrm{~cm}$; radius: $3 \mathrm{~cm}$ ), of which 2 are placed along the axis and 12 along the radius, placed collinearly at each collimator. Simulation were performed to determine the overall efficiency (geometrical plus photopeak efficiency) when adopting two different types of detectors: HpGe vs. Lanthanum Bromide scintillators ( $\mathrm{LaBr} 3)$. We assumed an isotropic source placed in the center of the plasma chamber, emitting in the range $100 \mathrm{keV}$ to $2 \mathrm{MeV}$. More details are given in [12].

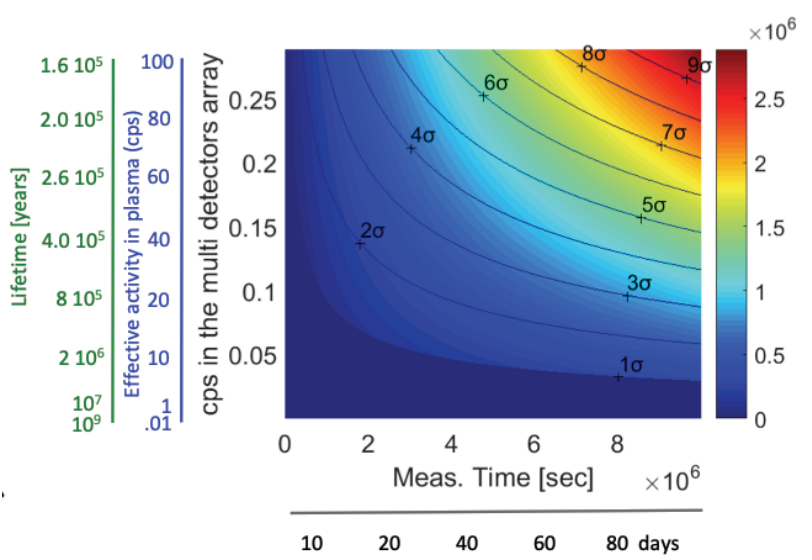

Fig. 4. Confidence levels plot showing the n-sigma level that is possible obtain after a given measurement time, reported in the $\mathrm{x}$ axis, vs the decay-rate (or the lifetime) of the Lutetium radionuclide.

The much higher energy resolution of HpGe detectors make these much more suitable for PANDORA than LaBr3. In the measurement, in fact, we have to include the strong plasmaself emitted background. An idea of time needed to get a certain n-sigma confidence level 
is illustrated in figure 4. In this plot we reported in the $\mathrm{x}$ axis, vs the decay-rate (or the lifetime) of the radionuclide. We considered the measurability of the physic case of Lutetium Lu, where a gamma at $306.78 \mathrm{keV}$ is emitted. Total number of $\gamma$-rays emitted after a given time can be estimated by eq. (3). In the green vertical axis is reported the lifetime expressed in years, starting from the lifetime of the neutral isotope to the values of lifetimes provided by the theory and that are feasible in our laboratory plasmas (variation about 6 order of magnitude for the case of Lu, for example). Considering these lifetimes, the effective activity in plasma (expressed in cps) are shown in the blue vertical axis and finally, taking into account the efficiency estimated by GEANT4 simulation, we obtained the cps detected in the multi detectors array (black vertical axis).

This plot summarizes the feasibility of the measurement and shows that for the variations of the lifetime expected from the theory in our laboratory plasmas, we expect that a measure lasting from tens of days to a couple of months is needed in order to obtain a 3 sigma level of confidence. Similar plots, for the other physics cases, have been performed.

\subsection{Plasma Diagnostics}

Conclusions coming from the figure 1 and equation 3 basically say us that we need to measure with a good precision plasma density and temperature, this way correlating them to eventual variations of the lifetime. Precise measurements of such plasma parameters, in addition, allow direct rescaling of measured lifetimes to astrophysical systems.

At INFN-LNS an arsenal of diagnostics tools is now available, including X-ray spectroscopy for soft and hard X-rays, space resolved soft-X ray spectroscopy by pin-hole cameras, RF probes and Spectrum Analyzers, Interferopolarimeters in the microwave Kband, Optical Emission Spectrometers [13]. The numbers obtained along the recent years say that now the plasma temperature and density can be measured - eventually in space and time resolved way - with an uncertainty that is less than $7 \%$ for the temperature, and around $25 \%$ for the total density.

Partial densities of warm and hot electrons are measured via X-ray spectroscopy with less than $10 \%$ uncertainty, while use of OES in the next future will allow to reduce density measurements uncertainties of at least a factor 2 .

\section{Conclusions}

An overview of the PANDORA project and its current status has been given. The project aims at measuring for the first time $\beta$-decays of astrophysical interest in magnetized plasmas. The setup is based on a B-minimum plasma trap surrounded by a $14 \mathrm{HpGe}$ detectors array. Construction of the trap is expected for the years 2020-2022, first measurements in 2023.

\section{References}

1. Litvinov Y. and Bosch F., Rep. Progr. Phys. 74, 016301 (2011)

2. Takahashi, et al., Phys Rev C 36, 1522 (1987)

3. Busso, M., Gallino, R., Wasserburg, G.J. 1999, Ann Rev. Astron. Astropys. 37, 239.

4. Cristallo, S. et al. 2011, Astrophys. J. Suppl. 197, 17.

5. Gallino, R. and Busso, M. 1986, in "From Nuclei to Stars". Proceedings of the International School of Physics "Enrico Fermi", Course XCI (North-Holland: Amsterdam). 
6. Raiteri, M. C., Busso, M., Gallino, R. and Picchio, G. 1991, ApJ 371, 665.

7. Gallino, R., Arlandini, C, Busso, M., et al, 1998, ApJ 497, 388.

8. Butcher, B. et al. 2013, J. Phys.: Conf. Ser. 420012141.

9. Butcher, X.D., et al. 2015, Phys. Rev. Lett, 114, 251102.

10. Takahashi, K. and Yokoi, K. 1983, ADNDT 36, 375

11. Dillman, I. et al. 2019, KADNIS version 1.0, in progress (https://expastro.de/kadonis1.0/).

12. E. Naselli et al. Nuclear $\beta$-dacays in plasmas: how to correlate plasma density and temperature, these proceedings

13. Mascali D. et al., EPJ A, 53, 145 (2017) 